\title{
Significant effects of biologic therapy on lipid profiles and insulin resistance in patients with rheumatoid arthritis
}

Der-Yuan Chen ${ }^{1,2,3,4^{*}}$, Yi-Ming Chen ${ }^{1,2,3 \dagger}$, Tsu-Yi Hsieh ${ }^{1 \dagger}$, Chia-Wei Hsieh ${ }^{1,2,3 \dagger}$, Chi-Chen Lin ${ }^{2,3}$ and Joung-Liang Lan ${ }^{5,6}$

\begin{abstract}
Introduction: The goal of this study was to investigate (1) the associations of rheumatoid arthritis (RA)-related inflammation or rheumatoid factor/anti-cyclic citrullinated peptide (anti-CCP) positivity with lipid profiles and insulin resistance (IR), (2) the effects of biologic therapy on lipid profiles and IR, and (3) potential predictors for the presence of subclinical atherosclerosis.
\end{abstract}

Methods: Serum levels of lipid profiles were determined by enzymatic methods in 32 adalimumab-treated patients, 16 etanercept-treated patients, 24 tocilizumab-treated patients, and 20 biologic-naïve patients. Atherogenic index, which corresponds to the ratio of total cholesterol to high-density lipoprotein cholesterol (HDL-C), was calculated. IR was measured by homeostasis model assessment. Pro-inflammatory cytokine levels were examined by enzyme-linked immunosorbent assay. Common carotid artery intima-media thickness was determined by using sonography.

Results: There was an inverse correlation between disease activity (disease activity score for 28 joints, or DAS28) and low-density lipoprotein cholesterol $(\mathrm{LDL}-\mathrm{C})$ levels $(r=-0.226, P<0.05)$ and a positive correlation between DAS28 and IR $(r=0.361, P<0.005)$. Anti-CCP-positive patients had significantly higher DAS28 and IR compared with anti-CCP-negative patients. There was also a positive correlation between IR and levels of interleukin- 6 or tumor necrosis factor-alpha (TNF-a). HDL-C levels significantly increased in patients receiving 6-month anti-TNF-a therapy, and levels of total cholesterol, LDL-C, and triglyceride increased in tocilizumab-treated patients. IR significantly decreased in patients under biologic therapy but was unchanged in biologic-naïve patients. Age, IR, and DAS28 were significant predictors of severe subclinical atherosclerosis (odds ratios of 1.08, 2.77, and 2.52, respectively).

Conclusions: Significant associations of RA-related inflammation with lipid profiles and IR indicate the involvement of RA in atherosclerosis pathogenesis. Biologic therapies were associated with IR reduction without change in atherogenic index, but their beneficial effects on atherosclerosis reduction need to be verified in the future.

\section{Introduction}

Rheumatoid arthritis (RA) is a chronic inflammatory articular disease $[1,2]$ that is complicated by accelerated atherosclerosis and subsequently leads to adverse cardiovascular $(\mathrm{CV})$ events [3,4]. Epidemiological studies have disclosed an increased risk of premature atherosclerosis

\footnotetext{
* Correspondence: dychen@vghtc.gov.tw

${ }^{\dagger}$ Equal contributors

'Division of Allergy, Immunology and Rheumatology, Taichung Veterans General Hospital and Faculty of Medicine, National Yang Ming University, Taiwan Boulevard, Taichung 40705, Taiwan

${ }^{2}$ Institute of Biomedical Science, National Chung Hsing University, No.250,

Guoguang Rd., South Dist., Taichung 40227, Taiwan

Full list of author information is available at the end of the article
}

and an increased mortality due to $\mathrm{CV}$ events in patients with RA [5-7].

Atherosclerosis-associated $\mathrm{CV}$ diseases (CVDs) are attributable to the traditional risk factors, including hypertension, dyslipidemia, diabetes mellitus (DM), and smoking in the general population [8,9]. A recent meta-analysis of traditional risk factors for CVD in patients with RA indicated an important role of low levels of high-density lipoprotein cholesterol (HDL-C) and an increased frequency of DM [10]. A nationwide cohort study demonstrates that RA is associated with the same risk of myocardial infarction as DM [11].

RA-related inflammation that is responsible for synovial lesions may be implicated in the development of 
accelerated atherosclerosis, leading to increased risk of CVD $[12,13]$. Furthermore, the magnitude and chronicity of inflammation strongly correlated with the emergence of premature atherosclerosis in RA [3,6,12,14]. The positivity of rheumatoid factor (RF) or anti-cyclic citrullinated peptide (anti-CCP) antibodies or both appears to be associated with high prevalence of subclinical atherosclerosis in RA [15]. In addition, the presence of HLA-DRB1*04 shared epitope alleles and tumor necrosis factor (TNF) A-308 (rs1800629) gene polymorphism is associated with a higher risk of CVD in patients with RA $[16,17]$.

Recent clinical studies identified elevated levels of proinflammatory cytokines, including TNF- $\alpha$ and interleukin- 6 (IL-6), as independent variables in association with arthrosclerosis in rheumatic patients and the general population $[13,14,18]$. TNF- $\alpha$ causes deterioration of the lipid profile and promotes insulin resistance (IR), both of which are traditional risk factors for atheroscerlosis $[14,18]$. Therefore, TNF- $\alpha$ inhibitors can induce favorable changes in lipid profiles with alteration of HDL composition [19]. Although previous studies failed to show that anti-TNF- $\alpha$ therapy could lower the risk of CVD [20,21], accumulating evidence suggests that TNF- $\alpha$ inhibitors can reduce the risk of future CV events in RA [22]. Besides the improvement of endothelial function [23], the possible mechanisms include a decrease of RA-associated inflammation, improvement of lipid profile [19], and the reduction of IR [24]. IL-6, a pro-inflammatory cytokine, may play a central role in decreasing total cholesterol (TC) levels and may also contribute to an increased IR in RA $[25,26]$. Tocilizumab, a humanized monoclonal antibody against IL-6 receptor (IL-6R), is effective in the treatment of RA $[27,28]$. Tocilizumab induced elevation of low-density lipoprotein cholesterol (LDL-C) but altered HDL particles toward an anti-inflammatory composition in RA [29]. These observations indicate that the reduction of RArelated inflammation and modulation of atherosclerosisassociated cytokines could be a potential strategy for the prevention of atherosclerosis in patients with RA.

Ultrasonography (US) of the carotid artery provides a non-invasive method for identifying atherosclerotic plaques, which reflect severe subclinical atherosclerosis and may predict the emergence of adverse CV events [30-33]. Common carotid artery intima-media thickness (ccIMT) measurements were shown to reflect the extent of coronary atherosclerosis [30,31]. Previous studies also showed that an increased ccIMT and evidence of plaques could predict the emergence of CVD in patients with RA [31,32]. Therefore, increased ccIMT or carotid plaques or both could be used as the gold standard for identification of severe subclinical atherosclerosis and patients at high risk of CVD [31-33].

The main objectives of this study were (1) to evaluate the associations of RA-related inflammation or RF/anti-
CCP positivity with serum levels of lipid profile, atherogenic index (AI), modified Framingham $\mathrm{CV}$ risk score (mFRS), IR, and pro-inflammatory cytokines; (2) to investigate the effects of biologic therapy on serum levels of lipid profiles, AI, mFRS, and IR in patients with RA; and (3) to examine the potential predictive factors and their optimal cutoff value for the presence of severe subclinical atherosclerosis.

\section{Methods \\ Patients}

In total, 92 consecutive biologic-naïve patients who fulfilled the 2010 revised criteria of the American College of Rheumatology for RA [34] were enrolled. Each of them had received traditional synthetic disease-modifying antirheumatic drugs (tsDMARDs), including methotrexate (MTX). Seventy-two patients started anti-TNF- $\alpha$ or anti-IL-6R therapy in combination with a stable dose of MTX 7.5 to $15 \mathrm{mg}$ weekly in accordance with the guidelines of the British Society for Rheumatology [35], whereas the other 20 patients continued with MTX therapy and other tsDMARDs (as the disease control). Patients with a recent history (within 1 year) of coronary heart disease or cerebral ischemic stroke were excluded from this study. Thirty-two patients received adalimumab at a dose of $40 \mathrm{mg}$ every other week, 16 patients received etanercept at a dose of $25 \mathrm{mg}$ twice weekly, and 24 patients received tocilizumab at a dose of $4 \mathrm{mg} / \mathrm{kg}$ once monthly during the first 3 months and then $8 \mathrm{mg} / \mathrm{kg}$ once monthly afterward, with concomitant MTX at a stable dose of 7.5 to $15 \mathrm{mg}$ weekly. The doses of tsDMARDs as well as oral corticosteroids remained unchanged and the use of intraarticular or parenteral corticosteroids was prohibited during the period of investigation. Disease activity was assessed by the 28-joint disease activity score (DAS28) at baseline and after 6 months of biologic or tsDMARDalone therapy, respectively [36]. The therapeutic response was evaluated after 6 months of therapy by using the European League Against Rheumatism (EULAR) response criteria [37]. We defined EULAR responders as patients with good and moderate EULAR therapeutic responses. The Institutional Review Board of Taichung Veterans General Hospital approved this study (CE12274), and the written consent of all participants was obtained in accordance with the Declaration of Helsinki.

\section{Measurements of serum levels of lipid profiles and atherogenic index}

All measurements were performed after an overnight fasting of 12 hours at baseline and after 6 months of biologic or tsDMARD-alone therapy, respectively. Serum levels of TC, triglyceride (TG), HDL-C, and LDL-C were measured by using enzymatic methods by a chemistry analyzer (Hitachi 7600; Hitachi, Tokyo, Japan) in 
accordance with the instructions of the manufacturer. The AI, which corresponds to the ratio of TC to HDL-C, was calculated.

\section{Measurements of the Framingham risk score and modified Framingham risk score}

FRS was determined on the basis of the demographic data and traditional risk factors [38]. The FRS estimates the 10-year risk of adverse CV events, including heart attack, stroke, or other occlusive arterial disease. Considering additional risks for patients with RA, we adopt a modified FRS (mFRS) by the application of a multiplier factor of 1.5 to those with two of the following three criteria: disease duration of more than 10 years, $\mathrm{RF}$ or anti-CCP antibody positivity, and the presence of extraarticular manifestations [39].

\section{Measurements of insulin resistance}

Serum insulin levels were determined by using a commercially available assay kit (IMMULITE, I-2000; EURO/ Diagnostic Products Cooperation, Gwynedd, UK). Homeostasis model assessment for IR (HOMA-IR) was calculated by using this formula: fasting plasma insulin (in microinternational units per liter) $\times$ fasting plasma glucose (in millimoles per liter)/22.5 [40].

\section{Ultrasound vascular imaging of carotid artery}

Ultrasound vascular imaging for carotid arteries included measurement of ccIMT and detection of focal plaques in the extracranial carotid tree. The final ccIMT was represented by the largest average ccIMT measured at the far wall of the common carotid arteries along a $10-\mathrm{mm}$ section of the artery proximal to the carotid bifurcation, and the proximal 15-mm-long segment of the internal and external carotid arteries. Plaque was defined as a localized thickening of more than $1.5 \mathrm{~mm}$ that did not uniformly involve the whole artery according to Mannheim consensus criteria [41]. A ccIMT of more than $0.90 \mathrm{~mm}$ or carotid plaques (or both) is defined as the gold standard for severe subclinical atherosclerosis [42].

\section{Determination of serum levels of pro-inflammatory cytokines}

Serum levels of TNF- $\alpha$ and IL- 6 were determined in 32 adalimumab-treated patients, 16 etanercept-treated patients, 14 tocilizumab-treated patients, and 10 biologicnaïve patients by using an enzyme-linked immunosorbent assay (PeproTech Inc., Rocky Hill, NJ, USA) in accordance with the instructions of the manufacturer.

\section{Statistical analysis}

Results are presented as the mean \pm standard deviation (SD) or standard error of mean (SEM). The nonparametric Kruskal-Wallis test was used for comparisons between groups. When this test showed a significant difference, the exact $P$ value was determined by using the Mann-Whitney $U$ test. The independent samples $t$ test was used for between-group (positivity and negativity for RF/anti-CCP antibodies) comparison of baseline levels of lipid profiles, AI, mFRS, IR, and pro-inflammatory cytokines. The non-parametric Spearman's correlations were determined between RA disease activity (DAS28) and levels of lipid profiles, AI, mFRS, IR, or pro-inflammatory cytokines. For comparison of levels of lipid profiles, AI, mFRS, IR, and pro-inflammatory cytokines during followup in RA patients before and after biologic therapy, the Wilcoxon signed rank test was employed. We also constructed a logistic regression model to evaluate the contribution of traditional risk factors and RA-related risk factors to the presence of severe subclinical atherosclerosis detected by US. The optimal cutoff values of age, AI, mFRS, IR, and DAS28 for the occurrence of severe subclinical atherosclerosis was determined by using receiver operating characteristic (ROC) curve analysis. The diagnostic sensitivity and specificity were determined by using MedCalc statistical software version 9.3 (MedCalc Software, Ostend, Belgium). A probability of less than 0.05 was considered significant.

\section{Results}

\section{Clinical characteristics of patients with rheumatoid} arthritis

As illustrated in Table 1, the majority of patients with RA were women and all patients had active disease (DAS28 of more than 3.2) at baseline. Among these patients, $78.3 \%$ were positive for RF and $69.6 \%$ were positive for anti-CCP antibodies; 58 (63.0\%) had a disease duration of at least 10 years; 13 (14.1\%) patients had extra-articular manifestations, including secondary Sjögren's syndrome in eight patients, interstitial lung disease in four, pleuritis/pericarditis in two, rheumatoid nodule in one, and rheumatoid vasculitis in one). According to the results of carotid US, $57.1 \%$ of patients with RA had severe subclinical atherosclerosis. There were no significant differences in the positive rate of RF/anti-CCP antibodies, daily dose of corticosteroids, weekly dose of MTX, or proportion of tsDMARDs used among four subgroups of RA patients at baseline.

After 6 months of biologic or tsDMARD-alone therapy, 25 (78.1\%) adalimumab-treated patients, 13 (81.3\%) etanercept-treated patients, and $20(83.3 \%)$ tocilizumabtreated patients were EULAR responders. However, only $50 \%$ of biologics-naïve patients were EULAR responders. The mean DAS28 changes ( \pm SDs) were $2.00 \pm 0.88$ in adalimumab-treated patients, $1.86 \pm 0.92$ in etanercepttreated patients, and $2.13 \pm 0.99$ in tocilizumab-treated patients, while a significantly lower change in DAS28 
Table 1 Demographic data and laboratory findings at baseline in four subgroups of rheumatoid arthritis patients according to therapeutic agents used

\begin{tabular}{lllll}
\hline & Adalimumab $(\mathbf{n}=\mathbf{3 2})$ & Etanercept $(\mathbf{n}=\mathbf{1 6})$ & Tocilizumab $\mathbf{( n = 2 4 )}$ & Without biologic $(\mathbf{n}=\mathbf{2 0})$ \\
\hline Mean age, years & $53.5 \pm 12.6$ & $54.4 \pm 7.8$ & $56.8 \pm 14.4$ & $57.0 \pm 11.4$ \\
Female proportion & $28(87.5 \%)$ & $13(81.3 \%)$ & $20(83.3 \%)$ & $17(85.0 \%)$ \\
Disease duration, years & $13.4 \pm 6.6$ & $13.6 \pm 9.0$ & $13.0 \pm 9.7$ & $12.3 \pm 5.8$ \\
Body mass index, kg/m ${ }^{2}$ & $24.8 \pm 4.6$ & $23.6 \pm 4.5$ & $24.5 \pm 3.7$ & $22.9 \pm 2.9$ \\
RF positivity & $28(87.5 \%)$ & $13(81.3 \%)$ & $17(70.8 \%)$ & $14(70.0 \%)$ \\
Anti-CCP positivity & $21(65.6 \%)$ & $11(68.8 \%)$ & $17(70.8 \%)$ & $15(75.0 \%)$ \\
Baseline ESR, mm/1st hour & $33.5 \pm 23.4$ & $39.0 \pm 36.0$ & $42.5 \pm 30.0$ & $35.8 \pm 22.1$ \\
Baseline DAS28 & $5.46 \pm 0.94$ & $5.48 \pm 0.98$ & $5.80 \pm 0.85$ & $5.12 \pm 0.59$ \\
Daily steroid dose, mg/day & $6.3 \pm 2.5$ & $6.9 \pm 1.9$ & $7.4 \pm 2.1$ & $7.0 \pm 2.2$ \\
DMARDs at baseline & & & $11.5 \pm 2.5$ & $11.3 \pm 2.8$ \\
\multicolumn{1}{c}{ MTX, weekly dose, mg } & $12.0 \pm 2.5$ & $10.6 \pm 3.7$ & $18(75.0 \%)$ & $17(85.0 \%)$ \\
Sulfasalazine & $27(84.4 \%)$ & $12(75.0 \%)$ & $19(79.2 \%)$ & $18(90.0 \%)$ \\
$\quad$ Hydroxychloroquine & $24(75.0 \%)$ & $12(75.0 \%)$ & $5(20.8 \%)$ & $7(35.0 \%)$ \\
Cyclosporine & $6(18.7 \%)$ & $4(25.0 \%)$ & $8(33.3 \%)$ & $8(40.0 \%)$ \\
Hypertension & $14(43.8 \%)$ & $6(37.5 \%)$ & $7(29.2 \%)$ & $6(30.0 \%)$ \\
Antihypertensive Rx & $8(25.0 \%)$ & $4(25.0 \%)$ & $1(4.2 \%)$ & $1(5.0 \%)$ \\
Diabetes mellitus & $2(6.2 \%)$ & $1(6.3 \%)$ & $0(0.0 \%)$ & $0(0.0 \%)$ \\
Hypoglycemic Rx & $1(3.1 \%)$ & $1(6.3 \%)$ & $3(12.5 \%)$ & $3(15.0 \%)$ \\
Current smoker & $3(9.4 \%)$ & $2(16.6 \%)$ &
\end{tabular}

Data are presented as mean \pm standard deviation or as number (percentage). anti-CCP, anti-cyclic citrullinated peptide (antibodies); DAS28, disease activity score for 28 joints; DMARDs, disease-modifying anti-rheumatic drugs; ESR, erythrocyte sedimentation rate; MTX, methotrexate; RF, rheumatoid factor; $\mathrm{Rx}$, treatment.

$(1.05 \pm 0.56)$ was observed in biologics-naïve patients $(P<0.001, P<0.01$, and $P<0.001$, respectively).

Associations of rheumatoid factor/anti-CCP antibody positivity with lipid profiles, atherogenic index, modified Framingham risk score, insulin resistance, and pro-inflammatory cytokines at baseline Compared with patients with negative RF, those with positive RF had significantly higher baseline DAS28 (mean \pm SEM, $5.63 \pm 0.11$ versus $5.13 \pm 0.17, P<0.01$ ), IR $(2.85 \pm 0.22$ versus $1.95 \pm 0.28, P<0.05)$, TNF- $\alpha$ levels $(285.61 \pm 87.78 \mathrm{pg} / \mathrm{mL}$ versus $48.19 \pm 8.03 \mathrm{pg} / \mathrm{mL}, P<0.05)$, and IL-6 levels $(734.16 \pm 245.38 \mathrm{pg} / \mathrm{mL}$ versus $230.28 \pm$ $54.94 \mathrm{pg} / \mathrm{mL}, P<0.01$ ) (Figure $1 \mathrm{~A}-\mathrm{C}$ ). Similarly, antiCCP-positive patients had significantly higher values in these parameters compared with anti-CCP-negative patients: baseline DAS28 (5.69 \pm 0.11 versus $5.00 \pm 0.13$, $P<0.01)$, IR ( $3.02 \pm 0.22$ versus $1.58 \pm 0.19, P<0.01)$, TNF$\alpha$ levels $(289.74 \pm 89.45 \mathrm{pg} / \mathrm{mL}$ versus $49.58 \pm 8.23 \mathrm{pg} / \mathrm{mL}$, $P<0.05)$, and IL-6 levels (748.48 \pm 250.21 versus $220.63 \pm$ 45.34, $P<0.01$ ) (Figure 1D-F). However, there were no significant differences in serum levels of lipid profile, AI, or mFRS between seropositive patients and seronegative patients.
Association of rheumatoid arthritis DAS28 with lipid profiles, atherogenic index, modified Framingham risk score, insulin resistance, DAS28, and pro-inflammatory cytokines at baseline

As shown in Figure 2, there was an inverse correlation between DAS28 and LDL-C levels and a positive correlation between DAS28 and IR, anti-CCP level, or levels of cytokines, including TNF- $\alpha$ and IL-6. However, there was no significant correlation between DAS28 and AI or mFRS.

\section{Correlation between cytokine levels and lipid profiles,} atherogenic index, modified Framingham risk score, or insulin resistance at baseline

There was an inverse correlation between IL-6 levels and LDL-C levels $(\mathrm{r}=-0.269, P<0.05)$ and a positive correlation between IL-6 levels and IR $(r=0.326, P<0.01)$. There was a positive correlation between TNF- $\alpha$ levels and IR $(r=0.416$, $P<0.005)$. However, there was no significant correlation between cytokine levels and lipid profiles or mFRS.

Change in lipid profiles, atherogenic index, modified Framingham risk score, and insulin resistance after therapy with or without biologics

Serum HDL-C levels significantly increased (mean \pm SEM, $71.29 \pm 2.24 \mathrm{mg} / \mathrm{dL}$ versus $75.40 \pm 2.88 \mathrm{mg} / \mathrm{mL}, P<0.05)$ 


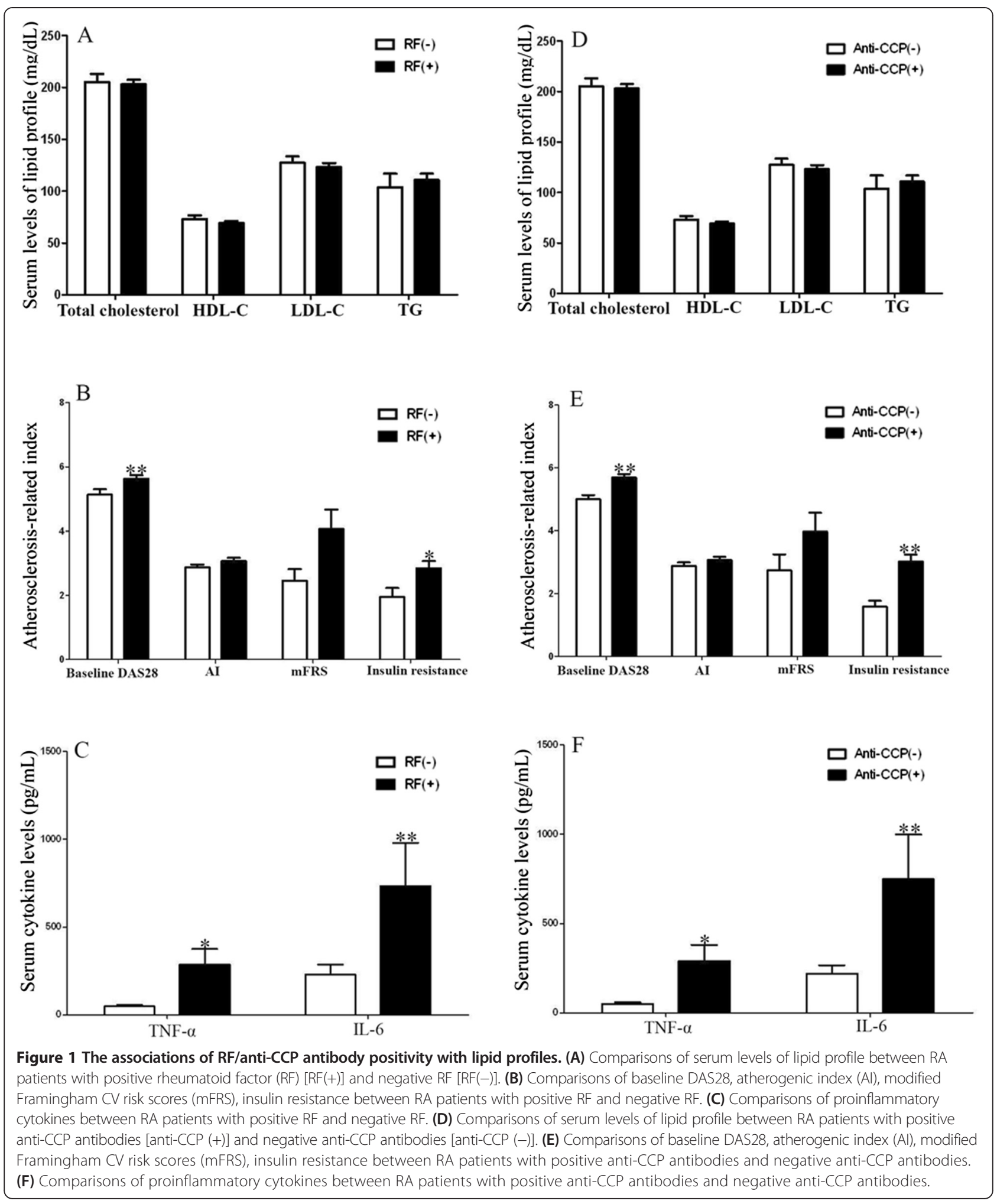

in patients receiving 6 months of anti-TNF- $\alpha$ therapy. As shown in Figure 3 and Table 2, there was no significant change in serum levels of lipid profiles, AI, or mFRS in patients receiving 6 months of adalimumab or etanercept.
Among tocilizumab-treated patients, levels of TC, LDL-C, and TG significantly increased without significant changes in HDL-C levels or AI. It is interesting that IR significantly decreased after 6 months of biologic therapy, including 

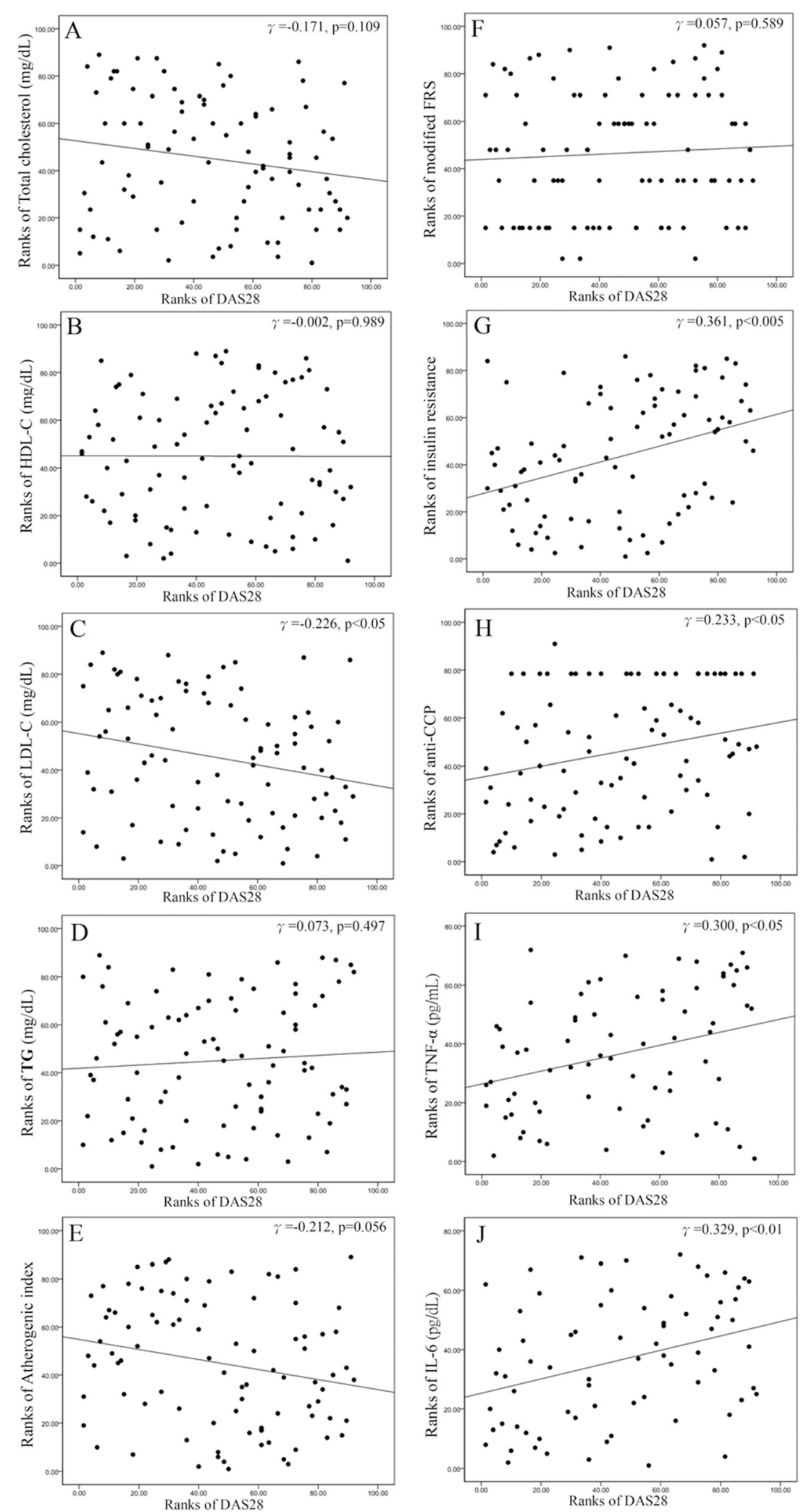

Figure 2 (See legend on next page.) 
(See figure on previous page.)

Figure 2 The association of disease activity (DAS28) with lipid profiles, Al, mFRS, IR, and proinflammatory cytokines. (A) The correlation between DAS28 and serum levels of total cholesterol. (B) The correlation between DAS28 and serum levels of HDL-C. (C) The correlation between DAS28 and serum levels of low-density LDL-C. (D) The correlation between DAS28 and serum levels of TG. (E) The correlation between DAS28 and atherogenic index (AI). (F) The correlation between DAS28 and modified Framingham CV risk scores (FRS). (G) The correlation between DAS28 and insulin resistance (IR). (H) The correlation between DAS28 and anti-CCP levels. (I) The correlation between DAS28 and serum levels of TNF-a. (J) The correlation between DAS28 and serum levels of IL-6.

adalimumab, etanercept, or tocilizumab. However, there was no significant change in levels of lipid profile, AI, mFRS, or IR in those not treated with biologic therapy.

\section{Logistic regression analysis}

As illustrated in Table 3, univariate regression analysis demonstrated that age, AI, mFRS, IR, disease duration of more than 10 years, and DAS28 were identified as potential predictors of severe subclinical atherosclerosis (odds ratio, $1.09, P<0.001 ; 2.12, P<0.05 ; 1.20, P<0.05 ; 2.43$, $P<0.001$; 3.33, $P<0.01$; and 2.94, $P<0.005$, respectively). To establish the best model to predict subclinical atherosclerosis, multivariate regression analysis was performed by choosing the variables that were significant by univariate regression analysis. Age, IR, and DAS28 were demonstrated as significant predictors of severe subclinical atherosclerosis (odds ratio, 1.08, $P<0.01 ; 2.77, P<0.005$; and $2.52, P<0.05$; respectively).

\section{Determination of the optimal cutoff values of predictive variables by receiver operating characteristic analysis}

The optimal cutoff value of age for predicting the occurrence of severe subclinical atherosclerosis was 59 years (area under ROC curve [AUC] of 0.784, sensitivity of $63.5 \%$, and specificity of $84.6 \%, P<0.001)$, the optimal cutoff value of AI was 3.02 (AUC of 0.654 , sensitivity of $55.8 \%$, and specificity of $71.8 \%, P<0.01)$, the optimal cutoff value of mFRS was 2.0 (AUC of 0.701 , sensitivity of $59.6 \%$, and specificity of $76.9 \%, P<0.001)$, the optimal cutoff value of IR was 1.27 (AUC of 0.791 , sensitivity of $94.2 \%$, and specificity of $53.9 \%, P<0.001)$, and the optimal cutoff value of DAS28 was 5.28 (AUC of 0.741 , sensitivity of $67.3 \%$, and specificity of $76.9 \%, P<0.001)$.

\section{Discussion}

In the present study, we demonstrated an inverse correlation between RA-related inflammation (DAS28) and serum LDL-C levels and a positive correlation between DAS28 and IR as well as levels of cytokines, including TNF- $\alpha$ and IL-6, at baseline. Moreover, patients who were seropositive for RF or anti-CCP antibodies had significantly higher DAS28, IR, TNF- $\alpha$ levels, and IL-6 levels compared with seronegative patients. After 6 months of biologic therapy, serum HDL-C levels significantly increased in patients receiving anti-TNF- $\alpha$ therapy, and levels of TC, LDL-C, and TG significantly increased in tocilizumab-treated patients. IR significantly decreased after 6 months of therapy with TNF- $\alpha$ inhibitors or IL$6 \mathrm{R}$ inhibitor, but AI did not show significant change. There was no significant change in levels of lipid profiles, AI, mFRS, or IR in patients who did not receive biologic therapy. In addition, multivariate regression analysis revealed that age, IR, and DAS28 were potential predictors of severe subclinical atherosclerosis.

Dyslipidemia is a well-established traditional risk factor for atherosclerosis $[4,9,10,43]$. Although the differences in the lipid profile between RA patients and the general population remain to be clarified, RA-related inflammation may be responsible for the change in lipid profiles [13-15,44]. Recent studies demonstrated that female patients with RA had significantly lower LDL-C levels than women in the general population $[45,46]$. Consistent with the findings of a previous study [44], our results showed an inverse correlation between RArelated inflammation (DAS28) and LDL-C levels in patients with RA. The lower LDL-C levels, in conjunction with elevated risk of CVDs in RA patients compared with the general population [5-7,46], support the hypothesis of a lipid paradox in this disease [44]. When compared with seronegative patients for RF or anti-CCP antibodies, our seropositive patients had significantly higher IR, consistent with a recent report that seropositivity is associated with IR in patients with inflammatory polyarthritis [47]. In agreement with a previous study that the presence of antiCCP antibodies appears to be associated with a high prevalence of subclinical atherosclerosis in RA [15], we demonstrated that patients with anti-CCP positivity had higher IR and DAS28, which have been implicated in the occurrence of atherosclerosis.

Accumulating evidence indicates that the accelerated atherosclerosis in RA cannot be explained by traditional risk factors alone [44]. Our results showed a positive correlation between RA disease activity (DAS28) and IR, which is an independent risk factor for atherosclerotic CVD [48]; these findings suggest that RA-related inflammation, as reflected by DAS28, is important in the emergence of IR. Our data also were consistent with the findings that patients with high-grade inflammation were more likely to have a high IR than those with low-grade inflammation [49]. In addition, pro-inflammatory cytokines, including TNF- $\alpha$ and IL-6, which are involved in RA pathogenesis, play a critical role in atherosclerosis in 

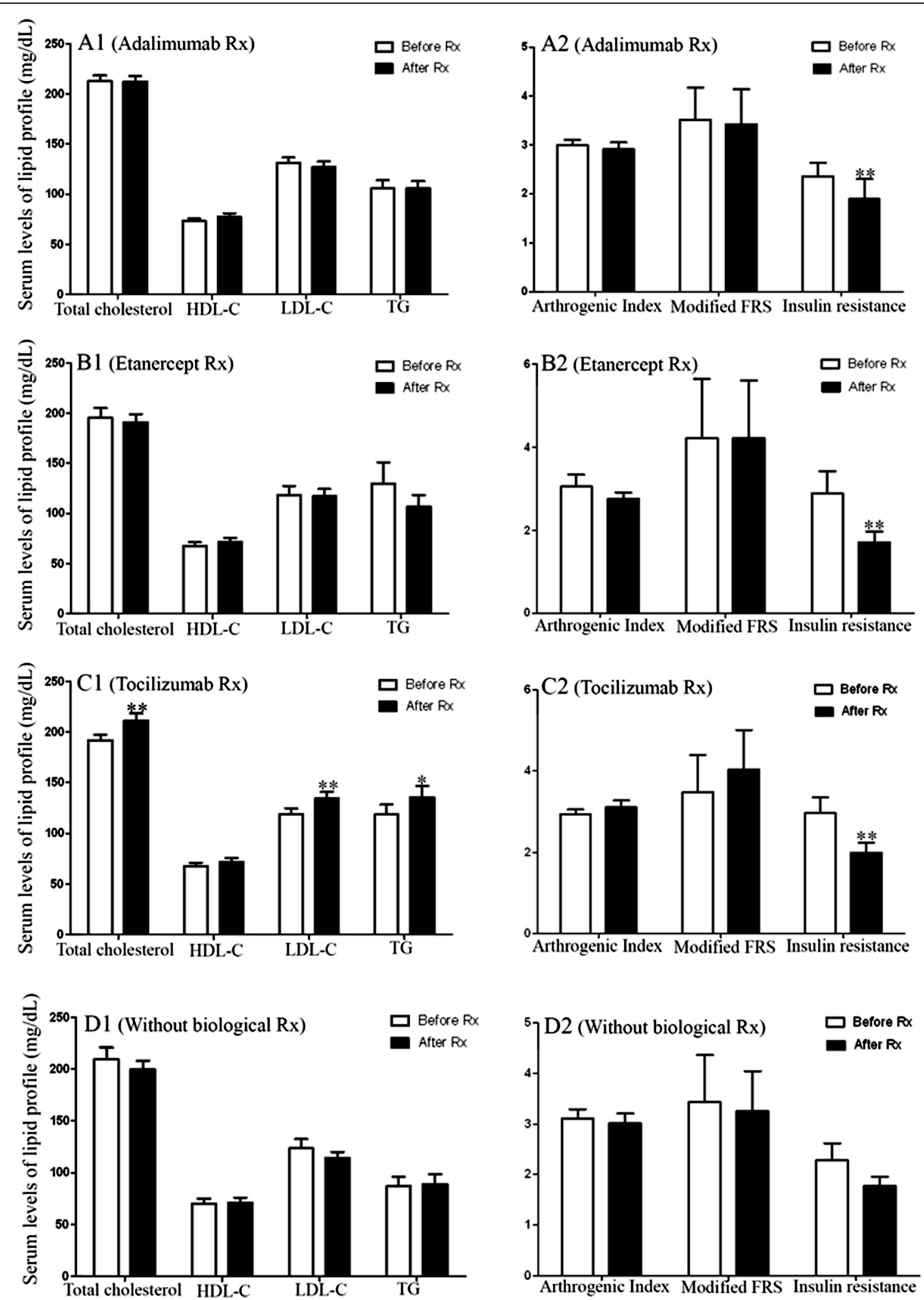

Figure $\mathbf{3}$ (See legend on next page.) 
(See figure on previous page.)

Figure $\mathbf{3}$ Change in lipid profiles, AI, mFRS, and IR after therapy with or without biologics. (A1) The changes in serum levels of lipid profile after 6 months of therapy in adalimumab-treated patients. (A2) The changes in AI, mFRS, and IR after 6 months of therapy in adalimumab-treated patients. (B1) The changes in serum levels of lipid profile after 6 months of therapy in etanercept-treated patients. (B2) The changes in Al, mFRS, and IR after 6 months of therapy in etanercept-treated patients. (C1) The changes in serum levels of lipid profile after 6 months of therapy in tocilizumab-treated patients. (C2) The changes in Al, mFRS, and IR after 6 months of therapy in tocilizumab-treated patients. (D1) The changes in serum levels of lipid profile after 6 months treatment in biologic-naïve patients. (D2) The changes in Al, mFRS, and IR after 6 months treatment in biologic-naïve patients.

patients with RA $[14,18]$. In the present study, we demonstrated that levels of TNF- $\alpha$ and IL- 6 were positively correlated with both DAS28 and IR in patients with RA. Our results were consistent with those of previous studies showing that TNF- $\alpha$ promotes IR $[14,18]$ and that IL-6 may contribute to an elevated IR [25,26]. Furthermore, our observations support the conclusion that RArelated inflammation and pro-inflammatory cytokines play an increasingly important role in the pathogenesis of IR in patients with RA [50].

During a longitudinal follow-up of patients with RA, we found that HDL-C levels were significantly increased in patients receiving 6 months of anti-TNF- $\alpha$ therapy, consistent with the results of previous studies $[19,51,52]$. In agreement with a systemic review with meta-analysis [53], our study showed no significant changes in LDL-C levels or AI in patients receiving 6 months of anti-TNF- $\alpha$ therapy. The findings of increases in levels of TC, LDL-C, and TG without apparent change in AI in our patients under anti-IL-6R therapies were consistent with previous reports $[28,29,54,55]$. These observations suggest that AI is less susceptible to the fluctuation of disease activity, making it more appropriate for predicting CVD risk in RA patients than individual lipid levels. Whether the changes in lipid profiles after biologic therapy contribute to CVD risk remains unclear.

It is interesting that there was a significant decrease in IR in patients receiving 6 months of anti-TNF- $\alpha$ therapy but that there was no significant change in IR in those receiving biologic-naïve therapies. Our results were similar to the findings of a study showing that knockout mice lacking TNF- $\alpha$ expression demonstrated improved IR [56],

Table 2 Change in lipid profiles, Al, mFRS, and IR in patients receiving biologic or biologic-naïve therapy

\begin{tabular}{|c|c|c|c|c|}
\hline & Adalimumab $(n=32)$ & Etanercept $(n=16)$ & Tocilizumab $(n=24)$ & Without biologic $(n=20)$ \\
\hline \multicolumn{5}{|c|}{ Total cholesterol, mg/dL } \\
\hline Baseline & $212.3 \pm 5.8$ & $195.3 \pm 9.8$ & $191.8 \pm 5.3$ & $209.6 \pm 11.2$ \\
\hline Week 24 & $212.1 \pm 5.6$ & $191.0 \pm 7.9$ & $211.5 \pm 6.8^{*}$ & $199.5 \pm 8.6$ \\
\hline \multicolumn{5}{|c|}{$\mathrm{HDL}-\mathrm{C}, \mathrm{mg} / \mathrm{dL}$} \\
\hline Baseline & $73.0 \pm 2.7$ & $67.5 \pm 4.1$ & $67.7 \pm 3.3$ & $70.4 \pm 4.4$ \\
\hline Week 24 & $77.1 \pm 3.8$ & $71.7 \pm 4.0$ & $72.1 \pm 4.0$ & $70.8 \pm 5.2$ \\
\hline \multicolumn{5}{|l|}{ LDL-C, mg/dL } \\
\hline Baseline & $131.3 \pm 5.3$ & $118.4 \pm 8.9$ & $119.2 \pm 5.5$ & $123.4 \pm 9.1$ \\
\hline Week 24 & $127.0 \pm 5.6$ & $117.0 \pm 7.4$ & $134.3 \pm 6.5^{*}$ & $114.2 \pm 6.0$ \\
\hline \multicolumn{5}{|c|}{ Triglyceride, mg/dL } \\
\hline Baseline & $105.6 \pm 8.3$ & $129.8 \pm 21.1$ & $118.8 \pm 9.8$ & $86.9 \pm 9.3$ \\
\hline Week 24 & $105.7 \pm 7.1$ & $106.4 \pm 12.0$ & $135.5 \pm 11.4^{*}$ & $88.9 \pm 9.6$ \\
\hline \multicolumn{5}{|c|}{ Atherogenic index } \\
\hline Baseline & $3.00 \pm 0.11$ & $3.05 \pm 0.29$ & $2.94 \pm 0.12$ & $3.11 \pm 0.18$ \\
\hline Week 24 & $2.91 \pm 0.14$ & $2.75 \pm 0.16$ & $3.11 \pm 0.16$ & $3.01 \pm 0.20$ \\
\hline \multicolumn{5}{|l|}{ Modified FRS } \\
\hline Baseline & $3.52 \pm 0.65$ & $4.22 \pm 1.44$ & $3.48 \pm 0.91$ & $3.43 \pm 0.93$ \\
\hline Week 24 & $3.42 \pm 0.72$ & $4.22 \pm 1.40$ & $4.04 \pm 0.97^{*}$ & $3.25 \pm 0.79$ \\
\hline \multicolumn{5}{|c|}{ Insulin resistance } \\
\hline Baseline & $2.75 \pm 0.29$ & $2.90 \pm 0.53$ & $2.97 \pm 0.38$ & $2.28 \pm 0.34$ \\
\hline Week 24 & $1.76 \pm 0.39^{*}$ & $1.71 \pm 0.26^{* *}$ & $1.99 \pm 0.25^{* *}$ & $1.77 \pm 0.18$ \\
\hline
\end{tabular}

Data are presented as mean \pm standard error of mean. ${ }^{*} P<0.05,{ }^{* *} P<0.005$, versus before treatment, determined by Wilcoxon signed rank test. FRS, Framingham risk score; HDL-C, high-density lipoprotein cholesterol; LDL-C, low-density lipoprotein cholesterol. 
Table 3 Univariate and multivariate association of traditionalcardiovascular risk factors and RA-rated factors with severe subclinical atherosclerosis

\begin{tabular}{|c|c|c|c|}
\hline Risk factors (univariate) & Odds ratio & $\begin{array}{l}95 \% \text { confidence } \\
\text { interval }\end{array}$ & $P$ value \\
\hline Age & 1.09 & $1.04-1.14$ & 0.000 \\
\hline Sex (female) & 0.89 & $0.18-4.39$ & 0.883 \\
\hline Smoking status & 3.87 & $0.79-19.06$ & 0.096 \\
\hline Hypertension Rx. & 1.35 & $0.55-3.32$ & 0.517 \\
\hline Body mass index & 0.95 & $0.86-1.05$ & 0.328 \\
\hline Total cholesterol & 1.00 & $0.98-1.01$ & 0.491 \\
\hline $\mathrm{HDL}-\mathrm{C}$ & 1.01 & $0.98-1.04$ & 0.467 \\
\hline LDL-C & 1.00 & $0.98-1.01$ & 0.511 \\
\hline Total triglyceride & 1.00 & $0.99-1.02$ & 0.640 \\
\hline Artherogenic index & 2.12 & $1.05-4.25$ & 0.035 \\
\hline Modified FRS & 1.20 & $1.01-1.42$ & 0.033 \\
\hline Insulin resistance & 2.43 & $1.50-3.93$ & 0.000 \\
\hline Disease duration $>10$ years & 3.33 & $1.36-8.15$ & 0.008 \\
\hline RF positivity & 1.41 & $0.44-4.44$ & 0.562 \\
\hline Anti-CCP positivity & 1.44 & $0.89-2.35$ & 0.142 \\
\hline TNF-a levels & 1.00 & $0.99-1.00$ & 0.743 \\
\hline IL-6 levels & 1.00 & $1.00-1.00$ & 0.196 \\
\hline DAS28 & 2.94 & $1.58-5.49$ & 0.001 \\
\hline Risk factors (multivariate) & Odds ratio & $\begin{array}{l}95 \% \text { confidence } \\
\text { interval }\end{array}$ & $P$ value \\
\hline Age & 1.08 & $1.02-1.15$ & 0.007 \\
\hline Artherogenic index & 1.31 & $0.98-1.76$ & 0.073 \\
\hline Modified FRS & 1.17 & $0.97-1.40$ & 0.093 \\
\hline Insulin resistance & 2.77 & $1.40-5.46$ & 0.003 \\
\hline Disease duration $>10$ years & 5.89 & $0.93-37.16$ & 0.059 \\
\hline DAS28 & 2.52 & $1.00-6.35$ & 0.049 \\
\hline
\end{tabular}

Anti-CCP, anti-cyclic citrullinated peptide (antibodies); DAS28, disease activity score for 28-joints; FRS, Framingham risk score; HDL-C, high-density lipoprotein cholesterol; IL-6, interleukin-6; LDL-C, low-density lipoprotein cholesterol; $\mathrm{RF}$, rheumatoid factor; Rx, treatment; TNF-a, tumor necrosis factor-alpha.

and they were consistent with recent studies showing beneficial effects of anti-TNF- $\alpha$ therapy on IR [24,52]. However, Rosenvinge et al. reported no significant change in IR from baseline in nine RA patients after 2 months of adalimumab therapy [57]. The discrepancy may be caused by the small number of subjects and a short-term therapy that was not long enough to detect treatment-related changes in IR. Similarly, we demonstrated a significant decrease in IR in patients receiving 6 months of anti-IL-6R (tocilizumab) therapy. Our results were consistent with a recent study that tocilizumab therapy decreases IR in patients with RA [58]. These observations imply that biologic therapies that block TNF- $\alpha$ or IL- 6 reduce IR, which is critical in the development of atherosclerosis in patients with RA.
To identify potential predictors for the occurrence of severe subclinical atherosclerosis [42], a logistic regression analysis was performed. Univariate analysis demonstrated that age, AI, mFRS, IR, disease duration of more than 10 years, and DAS28 were significant predictors of severe subclinical atherosclerosis. Our results supported previous studies revealing that age, AI, and FRS at baseline were associated with the occurrence of subclinical atherosclerosis in patients with RA [59,60]. Our data were also similar to another study reporting that IR was an individual predictor for identifying subjects with carotid plaque [61]. Disease duration of more than 10 years was a significant predictor of subclinical atherosclerosis in this study resonated with previous findings that a higher ccIMT was observed in RA patients with longer disease duration [62], and supported the claim that RA patients with disease duration of more than 10 years would need to undergo carotid US [63]. DAS28 was also a significant predictor of subclinical atherosclerosis, consistent with the findings of previous studies showing that RA-related inflammation is implicated in the development of atherosclerosis $[12,13]$. Similar to the results of other studies [15,64], we demonstrated a trend of an association of anti-CCP positivity with the occurrence of subclinical atherosclerosis $(\mathrm{p}=0.142)$. However, our findings should be verified by further extensive studies because of the use of a large number of variates in this small sample of patients.

Selection of the optimal cutoff value of age, AI, mFRS, IR, and DAS28 at baseline for predicting severe subclinical atherosclerosis may have clinical implications. Using ROC analysis, we demonstrated that patients with age above 59 years, AI above 3.02, mFRS above 2.0, IR above 1.27, and DAS28 above 5.28 might have a high probability of developing severe subclinical atherosclerosis. However, our results are preliminary; hence, the use of these cutoff values in clinical practice cannot be recommended until their external validity has been confirmed.

Some limitations in this study should be addressed. This is a preliminary study that enrolled a limited number of active RA patients who were followed up for 6 months. Actual CV events and mortality were not available, and positive results of carotid US were used as a surrogate marker of severe subclinical atherosclerosis. In consideration of the effects of corticosteroids and multiple DMARDs on levels of lipid profile, we did not analyze the differences in baseline levels of lipid profiles between RA patients and healthy subjects. However, recent studies and a meta-analysis have demonstrated the differences in lipid profiles between RA patients and a healthy population $[10,45,46]$. In addition, none of the enrolled patents in our study was in an early stage of RA and this may limit the generalizability of these results to the whole population. A long-term study enrolling 
larger groups of RA patients, including an early RA population, who receive biologic therapy and tsDMARDs alone is required to confirm these data. Because adipokines are of main relevance in the development of the metabolic syndrome frequently observed in patients with RA, the investigation of the effects of biologic therapy on adipokines [65] would be needed. Finally, qualitative changes in lipid profile such as HDL-C subfractions [66] and small dense LDL-C particles were not addressed.

\section{Conclusions}

Our results show significant associations of RA-related inflammation with LDL-C levels and IR. Patients with seropositive RA had significantly higher DAS28, IR, and levels of pro-inflammatory cytokines at baseline when compared with seronegative patients. Biologic therapies are associated with a significant increase in HDL-C levels (TNF- $\alpha$ inhibitors) or levels of TC, LDL-C, and TG (IL-6R inhibitor) without apparent change in AI. Biologic therapies could also improve insulin sensitivity. Age, AI, mFRS, IR, long disease duration, and DAS28 could predict the emergence of subclinical atherosclerosis in patients with RA. Early identification of traditional risk factors, tight control of RA-related inflammation with biological therapy, and ongoing monitoring of CVD risk factors are mandatory for slowing the progression of atherosclerosis in patients with RA.

\begin{abstract}
Abbreviations
Al: atherogenic index; anti-CCP: anti-cyclic citrullinated peptide; AUC: area under receiver operating characteristic curve; cCIMT: common carotid artery intima-media thickness; CV: cardiovascular; CVD: cardiovascular disease; DAS28: 28-joint disease activity score; DM: diabetes mellitus; DMARD: disease-modifying anti-rheumatic drug; EULAR: European League Against Rheumatism; FRS: Framingham risk score; HDL-C: high-density lipoprotein cholesterol; IL-6: interleukin-6; IL-6R: interleukin-6 receptor; IR: insulin resistance; LDL-C: low-density lipoprotein cholesterol; mFRS: modified Framingham risk score; MTX: methotrexate; RA: rheumatoid arthritis; RF: rheumatoid factor; ROC: receiver operating characteristic; SD: standard deviation; SEM: standard error of the mean; TC: total cholesterol; TG: triglyceride; TNF: tumor necrosis factor; tsDMARD: traditional synthetic disease-modifying anti-rheumatic drug; US: ultrasonography.
\end{abstract}

\section{Competing interests}

The authors declare that they have no competing interests.

\section{Authors' contributions}

D-YC conceived of the study, generated the original hypothesis, designed the study, acquired clinical data, conducted the analysis of data, and drafted and revised the manuscript. Y-MC, T-YH, and C-WH contributed equally to this work, performed clinical assessment, acquired clinical data, conducted the analysis of data, and revised the manuscript. C-CL and J-LL performed data analysis and revised the manuscript. All authors read and approved the final manuscript for publication.

\section{Acknowledgments}

This work was supported by a grant (NSC-102-2321-B-010-016) from the National Science Council, Taiwan, and by a grant (TCVGH 1023802C) from Taichung Veterans General Hospital. We thank the Biostatistics Task Force of Taichung Veterans General Hospital.

\section{Author details}

'Division of Allergy, Immunology and Rheumatology, Taichung Veterans General Hospital and Faculty of Medicine, National Yang Ming University, Taiwan Boulevard, Taichung 40705, Taiwan. ${ }^{2}$ Institute of Biomedical Science, National Chung Hsing University, No.250, Guoguang Rd., South Dist., Taichung 40227, Taiwan. ${ }^{3}$ Rong Hsing Research Center for Translational Medicine, National Chung Hsing University, No.250, Guoguang Rd., South Dist., Taichung 40227, Taiwan. ${ }^{4}$ Institute of Microbiology and Immunology, Chung Shan Medical University, No.110, Sec.1, Jianguo N.Rd., Taichung 40201, Taiwan. ${ }^{5}$ Division of Immunology and Rheumatology, China Medical University Hospital, No. 2, Yude Rd., North Dist., Taichung 40404, Taiwan. ${ }^{6}$ College of Medicine, China Medical University, No. 2, Yude Rd., North Dist., Taichung 40404, Taiwan.

Received: 6 August 2014 Accepted: 16 February 2015 Published online: 07 March 2015

\section{References}

1. Choy EH, Panayi GS. Cytokine pathways and joint inflammation in rheumatoid arthritis. N Engl J Med. 2001;344:907-16.

2. Firestein GS. Evolving concepts of rheumatoid arthritis. Nature 2003:423:356-61.

3. Gonzalez-Gay MA, Gonzalez-Juanatey C, Pineiro A, Garcia-Porrua C, Testa A, Llorca J. High-grade C-reactive protein elevation correlates with accelerated atherogenesis in patients with rheumatoid arthritis. J Rheumatol. 2005;32:1219-23.

4. Södergren A, Karp K, Boman K, Eriksson C, Lundström E, Smedby T, et al. Atheroslcerosis in early rheumatoid arthritis: very early endothelial activation and rapid progression of intima media thickness. Arthritis Res Ther. 2010;12:R158.

5. Symmons DP, Gabriel SE. Epidemiology of CVD in rheumatic disease, with a focus on RA and SLE. Nat Rev Rheumatol. 2011;31:399-408.

6. Chung CP, Oeser A, Raggi P, Gebretsadik T, Shintani AK, Sokka T, et al, Increased coronary-artery atherosclerosis in rheumatoid arthritis: relationship to disease duration and cardiovascular risk factors. Arthritis Rheum. 2005;52:3045-53

7. Yiu KH, Tse HF, Mok MY, Lau CS. Ethnic differences in cardiovascular risk in rheumatic disease: focus on Asians. Nat Rev Rheumatol. 2011;7:609-18.

8. Expert Panel on Detection, Evaluation, and Treatment of High Blood Cholesterol in Adults. Executive summary of the third report of the National Cholesterol Education Program (NCEP) Expert Panel on Detection, Evaluation, and Treatment of High Blood Cholesterol in Adults (Adult Treatment Panel III). JAMA. 2001;285:2486-97.

9. D'Agostino Sr RB, Vasan RS, Pencina MJ, Wolf PA, Cobain M, Massaro JM, et al. General cardiovascular risk profile for use in primary care: the Framingham Heart Study. Circulation. 2008;117:743-53.

10. Boyer JF, Gourraud PA, Cantagrel A, Davignon JL, Constantin A. Traditional cardiovascular risk factors in rheumatoid arthritis: a meta-analysis. Joint Bone Spine. 2011;78:179-83.

11. Lindhardsen J, Ahlehoff $\mathrm{O}$, Gislason GH, Madsen OR, Olesen JB, Torp-Pedersen C, et al. The risk of myocardial infarction in rheumatoid arthritis and diabetes mellitus: a Danish nationwide cohort study. Ann Rheum Dis. 2011;70:929-34.

12. Sattar N, McCarey DW, Capell H, Mclnnes IB. Explaining how "highgrade" systemic inflammation accelerates vascular risk in rheumatoid arthritis. Circulation. 2003;108:2957-63.

13. McEntegart A, Capell HA, Creran D, Rumley A, Woodward M, Lowe GD. Cardiovascular risk factors including thrombotic variables in a population with rheumatoid arthritis. Rheumatology (Oxford). 2001:40:640-4.

14. Rho YH, Chung CP, Oeser A, Solus J, Asanuma Y, Sokka T, et al. Inflammatory mediators and premature coronary atherosclerosis in rheumatoid arthritis. Arthritis Rheum. 2009;61:1580-5.

15. Gerli R, Bocci EB, Sherer Y, Vaudo G, Moscatelli S, Shoenfeld Y. Association of anti-cyclic citrullinated peptide antibodies with subclinical atherosclerosis in patients with rheumatoid arthritis. Ann Rheum Dis. 2008;67:724-5.

16. Gonzalez-Gay MA, Gonzalez-Juanatey C, Lopez-Diaz MJ, Piñeiro A, Garcia-Porrua C, Miranda-Filloy JA, et al. HLA-DRB1 and persistent chronic inflammation contribute to cardiovascular events and cardiovascular mortality in patients with rheumatoid arthritis. Arthritis Rheum. 2007;57:125-32.

17. Rodríguez-Rodríguez L, González-Juanatey C, Palomino-Morales R, Vázquez-Rodríguez TR, Miranda-Filloy JA, Fernández-Gutiérrez B, et al. 
TNFA -308 (rs1800629) polymorphism is associated with a higher risk of cardiovascular disease in patients with rheumatoid arthritis. Atherosclerosis. 2011;216:125-30.

18. Haddy N, Sass C, Droesch S, Zaiou M, Siest G, Ponthieux A, et al. IL-6, TNF-a and atherosclerosis risk indicators in healthy family population: the STANISLAS cohort. Atheroslcerosis. 2003;170:277-83.

19. van Eijk IC, de Vries MK, Levels JHM, Peters MJ, Huizer EE, Dijkmans BA, et al. Improvement of lipid profile is accompanied by atheroprotective alterations in high-density lipoprotein composition upon tumor necrosis factor blockade: a prospective cohort study in ankylosing spondylitis. Arthritis Rheum. 2009;60:1324-30.

20. Ljung L, Simard JF, Jacobsson L, Rantapää-Dahlqvist S, Askling J, Anti-Rheumatic Therapy in Sweden (ARTIS) Study Group. Treatment with tumor necrosis factor inhibitors and the risk of acute coronary syndromes in early rheumatoid arthritis. Arthritis Rheum. 2012;64:42-52.

21. Barnabe C, Martin BJ, Ghali WA. Systematic review and meta-analysis: anti-tumor necrosis factor alpha therapy and cardiovascular events in rheumatoid arthritis. Arthritis Care Res. 2011;63:522-9.

22. Westlake SL, Colebatch AN, Baird J, Curzen N, Kiely P, Quinn M, et al. Tumour necrosis factor antagonists and the risk of cardiovascular disease in patients with rheumatoid arthritis: a systematic literature review. Rheumatology (Oxford). 2011;50:518-31.

23. Gonzalez-Juanatey C, Llorca J, Sanchez-Andrade A, Garcia-Porrua C, Martin J, Gonzalez-Gay MA. Short-term adalimumab therapy improves endo-thelial function in patients with rheumatoid arthritis refractory to infliximab. Clin Exp Rheumatol. 2006;24:309-12.

24. Gonzalez-Gay MA, Gonzalez-Juanatey C, Vazquez-Rodriguez TR, Miranda-Filloy JA, Llorca J. Insulin resistance in rheumatoid arthritis: the impact of the antiTNF-a therapy. Ann N Y Acad Sci. 2010;1193:153-9.

25. Abeywardena MY, Leifert WR, Warnes KE, Varghese JN, Head RJ. Cardiovascular biology of interleukin-6. Curr Pharm Des. 2009;15:1809-21.

26. Chung CP, Oeser A, Solus JF, Gebretsadik T, Shintani A, Avalos I, et al. Inflammation-associated insulin resistance: differential effects in rheumatoid arthritis and systemic lupus erythematosus define potential mechanisms. Arthritis Rheum. 2008;58:2105-12.

27. Yazici $Y$, Curtis JR, Ince $A$, Baraf $H$, Malamet $R L$, Teng $L L$, et al. Efficacy of tocilizumab in patients with moderate to severe active rheumatoid arthritis and a previous inadequate response to disease-modifying antirheumatic drugs: the ROSE study. Ann Rheum Dis. 2012;71:198-205.

28. Nishimoto N, Hashimoto J, Miyasaka N, Yamamoto K, Kawai S, Takeuchi T, et al. Study of Active Controlled Monotherapy Used for Rheumatoid Arthritis, an IL-6 inhibitor (SAMURAI): evidence of clinical and radiographic benefit from an X-ray reader-blinded randomized controlled trial of tocilizumab. Ann Rheum Dis. 2007;66:1162-7.

29. Mclnnes IB, Thompson L, Giles JT, Bathon JM, Salmon JE, Beaulieu AD, et al. Effect of interleukin-6 receptor blockade on surrogates of vascular risk in rheumatoid arthritis: MEASURE, a randomized, placebo-controlled study. Ann Rheum Dis. 2015;74:694-702

30. Nambi V, Chambless L, Folsom AR, He M, Hu Y, Mosley T, et al. Carotid intima-media thickness and presence or absence of plaque improves prediction of coronary heart disease risk: the ARIC (Atherosclerosis Risk In Communities) study. J Am Coll Cardiol. 2010;55:1600-7.

31. Gonzalez-Juanatey C, Llorca J, Martin J, Gonzalez-Gay MA. Carotid intima media thickness predicts the development of cardiovascular events in patients with rheumatoid arthritis. Semin Arthritis Rheum. 2009;38:366-71

32. Evans MR, Escalante A, Battafarano DF, Freeman GL, O'Leary DH, del Rincón I. Carotid atherosclerosis predicts incident acute coronary syndromes in rheumatoid arthritis. Arthritis Rheum. 2011;63:1211-20.

33. Polak JF, Pencina MJ, Pencina KM, O'Donnell CJ, Wolf PA, D'Agostino Sr RB. Carotid-wall intima-media thickness and cardiovascular events. N Engl J Med. 2011;365:213-21.

34. Aletaha D, Neogi T, Silman AJ, Funovits J, Felson DT, Bingham 3rd CO, et al. The 2010 rheumatoid arthritis classification criteria: an American College of Rheumatology/European League Against Rheumatism collaborative initiative. Ann Rheum Dis. 2010;69:1580-8.

35. Ledingham J, Deighton C. British Society for Rheumatology Standards, Guidelines and Audit Working Group. Update on the British Society for Rheumatology guidelines for prescribing TNFalpha blockers in adults with rheumatoid arthritis (update of previous guidelines of April 2001). Rheumatology (Oxford). 2005;44:157-63.
36. Prevoo ML. van 't Hof MA, Kuper $H H$, van Leeuwen MA, van de Putte LB, van Riel PL. Modified disease activity scores that include twenty-eight-joint counts. Development and validation in a prospective longitudinal study of patients with rheumatoid arthritis. Arthritis Rheum. 1995:38:44-8.

37. Van Gestel AM, Prevoo ML, van't Hof MA, van Rijswijk MH, van de Putte LB, van Riel PL. Development and validation of the European League Against Rheumatism response criteria for rheumatoid arthritis. Comparison with the preliminary American College of Rheumatology and the World Health Organization/International League Against Rheumatism Criteria. Arthritis Rheum. 1996;39:34-40.

38. Grundy SM, Pasternak R, Greenland P, Smith Jr S, Fuster V. Assessment of cardiovascular risk by use of multiple-risk-factor assessment equations: a statement for healthcare professionals from the American Heart Association and the American College of Cardiology. Circulation. 1999;100:1481-92.

39. Peters MJ, Symmons DP, McCarey D, Dijkmans BA, Nicola P, Kvien TK, et al. EULAR evidence-based recommendations for CV risk management in patients with rheumatoid arthritis and other forms of inflammatory arthritis. Ann Rheum Dis. 2010;69:325-31.

40. Matthews DR, Hosker JP, Rudenski AS, Naylor BA, Treacher DF, Turner RC Homeostasis model assessment: insulin resistance and beta-cell function from fasting plasma glucose and insulin concentrations in man. Diabetologia. 1985:28:412-9.

41. Touboul PJ, Hennerici MG, Meairs S, Adams H, Amarenco P, Bornstein N, et al. Mannheim carotid intima-media thickness consensus (2004-2006-2011). An update on behalf of the Advisory Board of the 3rd, 4th, and 5th Watching the Risk Symposium, 13th,15th, and 20th European Stroke Conferences, Mannheim, Germany, 2004, Brussels, Belgium, 2006, and Hamburg, Germany, 2011. Cerebrovasc Dis. 2012:34:290-6.

42. Corrales A, González-Juanatey C, Peiró ME, Blanco R, Llorca J, González-Gay MA. Carotid ultrasound is useful for the cardiovascular risk stratification of patients with rheumatoid arthritis: results of a population-based study. Ann Rheum Dis. 2014;73:722-7.

43. Castelli WP, Garrison RJ, Wilson PW, Abbott RD, Kalousdian S, Kannel WB. Incidence of coronary heart disease and lipoprotein cholesterol levels. The Framingham Study. JAMA. 1986;256:2835-8.

44. Myasoedova E, Crowson CS, Kremers HM, Roger VL, Fitz-Gibbon PD, Therneau TM, et al. Lipid paradox in rheumatoid arthritis: the impact of serum lipid measures and systemic inflammation on the risk of cardiovascular disease. Ann Rheum Dis. 2011;70:482-7.

45. Liao KP, Cai T, Gainer VS, Cagan A, Murphy SN, Liu C, et al. Lipid and lipoprotein levels and trend in rheumatoid arthritis compared to the general population. Arthritis Care Res. 2013;65:2046-50.

46. Myasoedova E, Crowson CS, Kremers HM, Fitz-Gibbon PD, Therneau TM, Gabriel SE. Total cholesterol and LDL levels decrease before rheumatoid arthritis. Ann Rheum Dis. 2010;69:1310-4.

47. Mirjafari H, Farragher TM, Verstappen SM, Yates A, Bunn D, Marshall T, et al Seropositivity is associated with insulin resistance in patients with early inflammatory polyarthritis: results from the Norfolk Arthritis Register (NOAR): an observational study. Arthritis Res Ther. 2011;13:R159.

48. Del Rincon I, Freeman GL, Haas RW, O'Leary DH, Escalante A. Relative contribution of cardiovascular risk factors and rheumatoid arthritis clinical manifestations to atherosclerosis. Arthritis Rheum. 2005;52:3413-23.

49. Dessein $\mathrm{PH}$, Joffe BI. Insulin resistance and impaired beta cell function in rheumatoid arthritis. Arthritis Rheum. 2006:54:2765-75.

50. Gonzalez-Gay MA, Gonzalez-Juanatey C, Martin J. Rheumatoid arthritis: a disease associated with accelerated atherogenesis. Semin Arthritis Rheum. 2005:35:8-17.

51. Spanakis E, Sidiropoulos P, Papadakis J, Ganotakis E, Katsikas G, Karvounaris $S$, et al. Modest but sustained increase of serum high density lipoprotein cholesterol levels in patients with inflammatory arthritis treated with infliximab. J Rheumatol. 2006:33:2440-6.

52. Stagakis I, Bertsias G, Karvounaris S, Kavousanaki M, Virla D, Raptopoulou A et al. Anti-tumor necrosis factor therapy improves insulin resistance, beta cell function, and insulin signaling in active rheumatoid arthritis patients with high insulin resistance. Arthritis Res Ther. 2012;14:R141.

53. Daïen Cl, Duny Y, Barnetche T, Daurès JP, Combe B, Morel J. Effect of TNF inhibitors on lipid profile in rheumatoid arthritis: a systemic review with meta-analysis. Ann Rheum Dis. 2012;71:862-8.

54. Georgiadis AN, Papavasiliou EC, Lourida ES, Alamanos Y, Kostara C, Tselepis $A D$, et al. Atherogenic lipid profile is a feature characteristic of patients with early rheumatoid arthritis: effect of early treatment-a prospective, controlled study. Arthritis Res Ther. 2006;8:R82. 
55. Popa CD, Arts E, Fransen J, van Riel PL. Atherogenic index and high-density lipoprotein cholesterol as cardiovascular risk determinants in rheumatoid arthritis: the impact of therapy with biologicals. Mediators Inflamm. 2012;2012:785946

56. Uysal KT, Wiesbrock SM, Marino MW, Hotamisligil GS. Protection from obesity-induced insulin resistance in mice lacking TNF-a function. Nature. 1997;389:610-4.

57. Rosenvinge A, Krogh-Madsen R, Baslund B, Pedersen BK. Insulin resistance in patients with rheumatoid arthritis: effect of anti-TNFalpha therapy. Scand J Rheumatol. 2007;36:91-6.

58. Schultz O, Oberhauser F, Saech J, Rubbert-Roth A, Hahn M, Krone W, et al. Effects of inhibition of interleukin-6 signaling on insulin sensitivity and lipoprotein (A) levels in human subjects with rheumatoid diseases. PLoS One. 2010;5:e14328.

59. Im CH, Kim NR, Kang JW, Kim JH, Kang JY, Bae GB, et al. Inflammatory burden interacts with conventional cardiovascular risk factors for carotid plaques formation in rheumatoid arthritis. Rheumatology (Oxford). 2014

60. Sandoo A, Chanchlani N, Hodson J, Smith JP, Douglas KM, Kitas GD. The relationship between cardiovascular disease risk prediction scores and vascular function and morphology in rheumatoid arthritis. Clin Exp Rheumatol. 2014;32:914-21.

61. Khalil A, Huffman MD, Prabhakaran D, Osmond C, Fall CH, Tandon N, et al. Predictors of carotid intima-media thickness and carotid plaque in young Indian adults: the New Delhi New birth cohort. Int J Cardiol. 2013;167:1322-8.

62. González-Juanatey C, Llorca J, González-Gay MA. Correlation between endothelial function and carotid atherosclerosis in rheumatoid arthritis patients with long-standing disease. Arthritis Res Ther. 2011;13:R101.

63. González-Gay MA, González-Juanatey C, Llorca J. Carotid ultrasound in the cardiovascular risk stratification of patients with rheumatoid arthritis: when and for whom? Ann Rheum Dis. 2012;71:796-8.

64. Gerli R, Sherer Y, Bocci EB, Vaudo G, Moscatelli S, Shoenfeld Y. Precocious atherosclerosis in rheumatoid arthritis: role of traditional and disease-related cardiovascular risk factors. Ann N Y Acad Sci. 2007:1108:372-81.

65. Gonzalez-Gay MA, Garcia-Unzueta MT, Gonzalez-Juanatey C, Miranda-Filloy JA, Vazquez-Rodriguez TR, De Matias JM, et al. Anti-TNF-alpha therapy modulates resistin in patients with rheumatoid arthritis. Clin Exp Rheumatol. 2008;26:311-6.

66. Arts E, Fransen J, Lemmers H, Stalenhoef A, Joosten L, van Riel P, et al. High-density lipoprotein cholesterol subfractions HDL2 and HDL3 are reduced in women with rheumatoid arthritis and may augment the cardiovascular risk of women with RA: a cross-sectional study. Arthritis Res Ther. 2012;14:R116.

\section{Submit your next manuscript to BioMed Central and take full advantage of:}

- Convenient online submission

- Thorough peer review

- No space constraints or color figure charges

- Immediate publication on acceptance

- Inclusion in PubMed, CAS, Scopus and Google Scholar

- Research which is freely available for redistribution 